\title{
Autopercepción de los Efectos en la Utilización de Dentífricos con Propuesta Aclaradora
}

\author{
Self-Perception of the Effects in the Use of Dentifrices With Clarifying Proposal
}

\author{
Ana Paula Gurgel Silva; Lucas Akccyl Albuquerque Alves; \\ Maria Arianny Costa Tavares \& Diala Aretha De Sousa Feitosa
}

SILVA, A. P. G.; ALVES, L. A. A.; TAVARES, M. A. C. \& FEITOSA, D. A. S. Autopercepción de los efectos en la utilización de dentífricos con propuesta aclaradora. Int. J. Odontostomat., 15(2):484-491, 2021.

RESUMEN: Las exigencias estéticas están aumentando en la sociedad, se sabe que la sonrisa es un factor determinante en la estética facial, y que los dientes blancos están asociados a signo de juventud y salud bucal. Actualmente, cremas dentales con propuesta blanqueador están al alcance de la mayoría de la población, lo que garantiza una mayor demanda de estos productos. Sin embargo, su efectividad aún genera dudas. La presente investigación tuvo como objetivo evaluar la práctica del cepillado con dentífricos que poseen propuesta blanqueadora, la satisfacción, la autopercepción de la respuesta aclaradora y la presencia de hipersensibilidad ocasionada durante su uso. Los datos fueron colectados por medio de cuestionarios, en los que fueron evaluados Pacientes $(n=50)$ atendidos en la Clínica Escuela del Centro Universitário Doutor Leão Sampaio, Académicos de Odontología $(n=50)$ del Centro Universitário Doutor Leão Sampaio y CirujanosDentistas $(n=50)$ actuantes de la ciudad de Juazeiro do Norte, Ceará. Se realizó análisis descriptivo de distribución de frecuencia, y se realizó la prueba de Qui-Cuadrado de Pearson, adoptándose un nivel de significancia del 5 \%. Se constató que todos los grupos ya han hecho o hacen uso de dentífricos con propuesta aclaradora. La propaganda influyó en el grupo de pacientes y académicos de odontología, sin embargo, para el grupo de los Cirujanos-Dentistas, ocurrió por conocimiento e influencia de otro profesional Cirujano-Dentista. La autopercepción fue negativa en cuanto a la eficacia blanqueador de estos productos, hubo interrupción, y las principales quejas fueron hipersensibilidad dentitaria y la eficacia blanqueadora, el uso de dentifrícios con propuesta aclaradora no fue recomendado por el grupo estudiado.

PALABRAS CLAVE: Estética, dentífricos, higiene oral, clareo dental.

\section{INTRODUCCIÓN}

La obtención de una sonrisa agradable y de una estética dental satisfactoria no es sólo una cuestión de vanidad, sino de necesidad en los días actuales, por los estándares de belleza impuestos por la sociedad. Muchos individuos, al no poseer esta apariencia deseada, pierden la autoestima, llevándolos a un comportamiento reservado y tímido, siendo que la mayoría de los pacientes insatisfechos con su sonrisa buscan modificarlo de alguna forma (Silva et al., 2015). Se sabe que el concepto de estética dental se trata de dientes blancos, bien alineados y contorneados, simétricos y proporcionales, conceptualizando una fusión de factores que reflejan además de una buena salud oral, status socioeconómico, jovialidad y expresividad (Feitosa et al., 2015).
Una de las principales causas de la insatisfacción en la estética de la sonrisa es el cambio de color de los dientes. Estos cambios pueden ser consecuencia de manchas extrínsecas, que se sitúan en la superficie del diente y pueden ser adquiridas por bacterias cromogénicas, humo, alimentos con pigmentos de colorantes (Bergesch et al., 2017). Por otro lado, las manchas intrínsecas están relacionadas con pigmentos que se localizan en íntimo contacto con el diente, pudiendo ser congénita o adquirida (Marson et al., 2005).

Actualmente el mercado comercializa diversas técnicas y productos en el intento de revertir la coloración de los dientes, entre ellos destacan, el aclaramiento casero y el de consultorio para dientes vitales 
y desvitalizados, el método de la cinta adhesiva que emplea el agente aclarador en bajas concentraciones (6\% a $10 \%)$, también la asociación de técnicas aclaradoras y la utilización de dentífricos con agentes con propuesta blanqueadora en su composición, como la sílice, bicarbonato de Calcio, Peróxido de Calcio, Peróxido de Urea entre otros vehículos que se insertan en la composición de estos (Lima et al., 2008).

El uso de dentífricos forma parte de los hábitos de higiene oral de la población mundial, auxilia en la limpieza de los dientes por medio de la remoción mecánica de residuos, pulido de la estructura dental, dificultando así la formación de la placa bacteriana y previniendo la caries y la enfermedad periodontal (Maden et al., 2018). En las últimas décadas, el papel de estos ha sido modificado, haciéndolos más especializados, actualmente están compuestos por sustancias que se presentan en forma de crema, pasta o gel en tubos convencionales que desempeñan las funciones de agente de pulido y terapéutico (Silva et al., 2009) como la inclusión del Flúor (anti-cariogénico), Nitrato de Plata (desensibilizante), Clorxidina (antibacteriano) y agentes abrasivos y peróxidos (agentes cosméticos).

Entre las diversas propuestas de tratamiento y materiales disponibles la única que puede ser administrada por el propio paciente de forma fácil y asequible es la utilización de dentífricos con propuesta aclaradora (Araújo et al., 2009). Estas características han estimulado la curiosidad de pacientes y profesionales en cuanto a su eficacia, además de su amplia difusión en los medios de comunicación, alcanzando todas las clases sociales, propiciando una popularización en la odontología estética (Monteiro et al., 2014).

El presente estudio tuvo como objetivo evaluar la autopercepción de Cirujanos Dentistas, pacientes atendidos en la Clínica Escuela del Centro Universitario Doctor León Sampaio y Académicos del curso de Odontología de esta misma institución, en cuanto a utilización, satisfacción, la autopercepción de la respuesta aclaradora y la presencia de hipersensibilidad ocasionada durante el uso de dentífricos con propuesta aclaradora.

\section{MATERIAL Y MÉTODO}

Se trata de un estudio observacional, transversal y descriptivo-analítico, realizado en la ciudad de Juazeiro do Norte (CE). La muestra fue compuesta por Académicos del curso de Odontología del Centro Universitário Doutor Leão Sampaio $(n=50)$, Pacientes atendidos en la Clínica Escuela de Odontología del Centro Universitário Doutor Leão Sampaio $(n=50)$ y Cirujanos-Dentistas que actúan en la ciudad de Juazeiro do Norte/CE $(n=50)$.

Para la realización de la investigación y recopilación de datos, se elaboró un cuestionario, abordando el uso de dentífricos con propuesta aclaradora, autopercepción de la propuesta aclaradora del producto utilizado y la sensibilidad durante el uso del dentífricos aclarador.

Esta encuesta fue aprobada por el Comité de Ética e Investigación. Los participantes fueron esclarecidos sobre la propuesta de la investigación y recibieron los Términos de Consentimiento Libre e Informado y el postesclarecido, para que luego pudieran participar de la investigación.

Para el análisis estadístico de los resultados, se ha elaborado una base de datos en el programa Statistical Package for the Social Sciences (SPSS) en la versión 22.0, en la que se realizó estadística descriptiva y elaboración de tablas cruzadas con las informaciones que describen las características de interés para la presente investigación. Se realizó la prueba de Qui-Cuadrado de Pearson para comprobar si existen diferencias estadísticamente significativas entre la recurrencia de las características estudiadas en función del perfil de los participantes del estudio, adoptando como criterio el valor de $p<0,05$ para indicar diferencias significativas.

\section{RESULTADOS}

De acuerdo con la recopilación de datos, los 150 participantes, proporcionalmente dividido entre Cirujanos Dentistas, Académicos de Odontología y Pacientes. En relación al uso de dentífricos con propuesta aclaradora, los resultados dispuestos en la Tabla I, muestran que $32(64,0 \%)$ de los Cirujanos-Dentistas relataron no utilizar, $27(54,0 \%)$ de los académicos respondieron que utilizan, y $28(56,0 \%)$ respondieron que también utilizan. Los resultados dispuestos en la Tabla II, muestran la indicación y si hubo interrupción del uso de dentífrico Clareador.

Entre los motivos relacionados con la interrupción del uso, fueron mencionados por los participantes 
de la investigación diversos motivos, que pueden ser observados en la Figura 1. Al utilizar el dentífrico, la mayoría citó que no hubo resultado blanqueador (32, $8 \%)$.

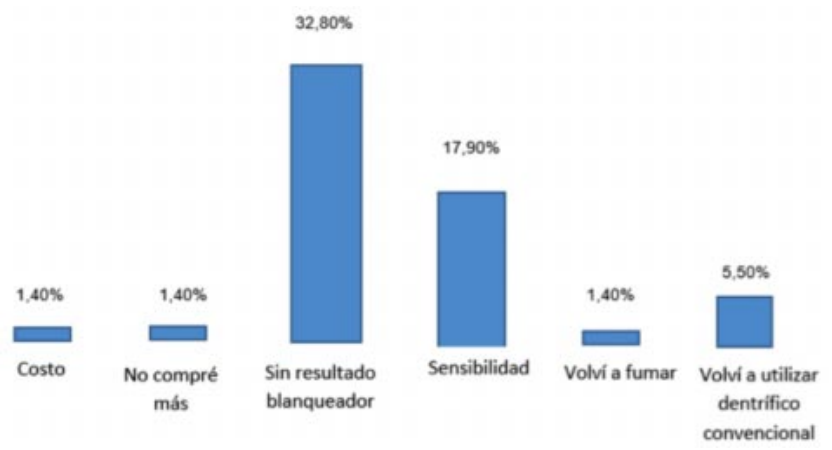

Fig. 1. Principales razones para la interrupción del cepillado con dentífrico aclarador.

Tabla I. Utilización de dentifrícios con propuesta aclaradora.

\begin{tabular}{lllll}
\hline \multirow{2}{*}{ Uso de Aclaramiento } & Sí & \multicolumn{3}{c}{ No } \\
& $\mathrm{N}$ & $\%$ & $\mathrm{~N}$ & $\%$ \\
\hline Cirujano-Dentista & 18 & 36,0 & 32 & 64,0 \\
Académico de Odontología & 27 & 54,0 & 23 & 46,0 \\
Paciente & 28 & 56,0 & 22 & 44,0 \\
$\quad$ Total & 73 & 48,7 & 77 & 51,3 \\
\hline
\end{tabular}

Se pudo verificar que la mayoría de los participantes no percibieron mejoras, no asociaron el dentífrico a otro tipo de aclaramiento, no recomendaría el producto y no sintieron sensibilidad (Tabla III).

En cuanto a la eficacia aclaradora de los dentífricos, la mayoría de los grupos de participantes, reportado no tener eficacia (Fig. 2).

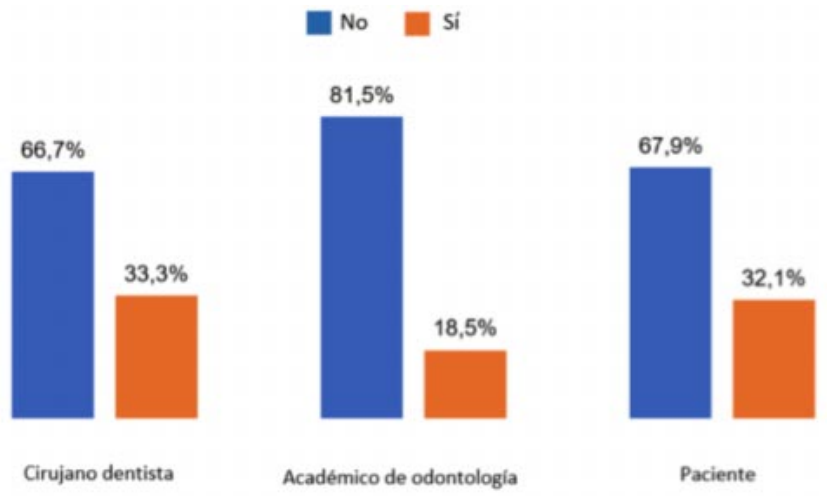

Fig. 2. Eficacia aclaradora de los dentifrícios con propuesta blanqueadora de los diferentes grupos investigados.

Tabla II. Indicación e interrupción del uso de dentifrício aclarador.

\begin{tabular}{|c|c|c|c|c|c|c|c|c|c|}
\hline \multirow[t]{2}{*}{ Variables } & \multirow[t]{2}{*}{ Categorías } & \multicolumn{2}{|c|}{ Cirujano- Dentis } & \multicolumn{2}{|c|}{$\begin{array}{c}\text { Académico de } \\
\text { Odontología }\end{array}$} & \multicolumn{2}{|c|}{ Paciente } & \multicolumn{2}{|c|}{ Total } \\
\hline & & $\bar{N}$ & $\%$ & $\mathrm{~N}$ & $\%$ & $\mathrm{~N}$ & $\%$ & $\bar{N}$ & $\%$ \\
\hline \multirow{2}{*}{ Indicación } & Cirujano- Dentista & 11 & 61,1 & 8 & 29,6 & 8 & 28,6 & 27 & 37 \\
\hline & Colegas o anuncios & 7 & 38,9 & 19 & 70,4 & 20 & 71,4 & 46 & 63 \\
\hline \multirow[t]{2}{*}{ Interrupción } & Sí & 8 & 44,4 & 19 & 70,4 & 18 & 64,3 & 45 & 38,4 \\
\hline & No & 10 & 55,6 & 8 & 29,6 & 10 & 35,7 & 28 & 61,6 \\
\hline
\end{tabular}

Tabla III. Aspectos observados en relación al uso de dentrífico con perspectiva del blanqueamiento y sensibilidad dental.

\begin{tabular}{|c|c|c|c|c|c|c|c|c|c|c|}
\hline \multirow[t]{2}{*}{ Variables } & \multirow[t]{2}{*}{ Categorías } & \multicolumn{2}{|c|}{$\begin{array}{l}\text { Cirujano- } \\
\text { Dentista }\end{array}$} & \multicolumn{2}{|c|}{$\begin{array}{l}\text { Ac adémico } \\
\text { Odontología }\end{array}$} & \multirow[t]{2}{*}{ de } & \multicolumn{2}{|c|}{ Paciente } & \multicolumn{2}{|c|}{ Total } \\
\hline & & $\mathbf{N}$ & $\%$ & $\mathbf{N}$ & $\%$ & & $\mathbf{N}$ & $\%$ & $\mathbf{N}$ & $\%$ \\
\hline \multirow[t]{2}{*}{ No tó Me joras } & Sí & 6 & 33,3 & 5 & 18,5 & & 5 & 17,9 & 16 & 21,9 \\
\hline & NO & 12 & 66,7 & 22 & 81,5 & & 23 & 82,1 & 57 & 78,1 \\
\hline \multirow[t]{2}{*}{ Uso Asociado } & sí & 4 & 22,2 & 3 & 11,1 & & 3 & 10,7 & 10 & 13,7 \\
\hline & NO & 14 & 77,8 & 24 & 88,9 & & 25 & 89,3 & 63 & 86,3 \\
\hline \multirow[t]{2}{*}{ Recomendaría } & sí & 6 & 33,3 & 5 & 18,5 & & 9 & 32,1 & 20 & 27,4 \\
\hline & NO & 12 & 66,7 & 22 & 81,5 & & 19 & 67,9 & 53 & 72,6 \\
\hline \multirow[t]{2}{*}{ Sintió Sensibilidad } & sí & 5 & 27,8 & 8 & 29,6 & & 12 & 42,9 & 25 & 34,2 \\
\hline & NO & 13 & 72,2 & 19 & 70,4 & & 16 & 57,1 & 48 & 65,8 \\
\hline \multirow{5}{*}{$\begin{array}{l}\text { Clasificación de la } \\
\text { sensibilidad* }\end{array}$} & 0 & 13 & 72,2 & 19 & 70,4 & & 16 & 57,1 & 48 & 65,8 \\
\hline & 1 & 1 & 5,6 & 3 & 11,1 & & 1 & 3,6 & 5 & 6,8 \\
\hline & 2 & 3 & 16,7 & 4 & 14,8 & & 5 & 17,9 & 12 & 16,4 \\
\hline & 3 & 1 & 5,6 & 1 & 3,7 & & 5 & 17,9 & 7 & 9,6 \\
\hline & 4 & 0 & 0,0 & 0 & 0,0 & & 1 & 3,6 & 1 & 1,4 \\
\hline
\end{tabular}




\section{DISCUSIÓN}

Una de las principales causas de insatisfacción en la estética de la sonrisa es el cambio en el color de los dientes (Briso et al., 2014; Soeteman et al., 2018). En los últimos años, los dientes con aspecto oscurecido se han convertido en una de las quejas más frecuentes de los pacientes (Marson et al.). Sin embargo, pueden revertirse cambios cromáticos severos modificando las características ópticas de los tejidos dentales, que pueden ser congénitas o adquiridas (Briso et. al.; Bergesch et al.), devolviendo la apariencia de una sonrisa blanca, saludable y dinámica a muchospacientes.

Esta preferencia asociada al gran atractivo por la estética en los medios de comunicación ha contribuido a aumentar la demanda de aclaramiento dental en los consultorios odontológicos (Menezes et al., 2003; Roopa et al., 2016). Este hecho los ha llevado a buscar además de consultorios odontológicos, para tratamientos aclaradores, productos con propuesta blanqueadora en el mercado, en busca de dientes cada vez más blancos (Antonini et al., 2007; Khamverdi et al., 2010; Melo et al., 2014; Pasquali et al., 2014; Abdelmegid et al., 2016; Bortolatto et al., 2016). Pues, además de las técnicas de aclaramiento realizadas o supervisadas por dentistas, como terapias de aclaramiento casero y en consultorio, existe un mercado creciente para productos de blanqueo dental que prometen blanqueo rápido y conveniente, incluyendo los dentífricos aclaradores de fácil acceso (Magalhães et al., 2011).

Las cremas dentales están al alcance de la población con costo asequible y la modificación de esos dentífricos generó un hito en la odontología cosmética y terapéutica (Silva et al., 2015), aunque efectos deletéreos puedan ocurrir, la literatura describe algunos daños a los tejidos duros dentales, incluida la disminución de la microdureza, el cambio de la rugosidad superficial y el aumento de la permeabilidad (Justin et al., 2019).

En este estudio, se constató que la mayoría de los académicos y pacientes utilizan dentífricos aclaradores en su higienización bucal, mientras que el $64 \%$ de los profesionales no hacen uso de este producto (Tabla I). No se observan diferencias estadísticamente significativas en la cantidad de investigados en relación al uso o no de dentífricos aclaradores, pero los Cirujanos-Dentistas hacen me- nor uso de los dentífricos con propuesta aclaradora, esto indica que los profesionales poseen un mayor conocimiento de los daños de estos productos en la estructura dental.

Se observan diferencias estadísticamente significativas en relación al tipo de fuente de indicación para el uso de los dentífricos blanqueadores. Entre los diferentes perfiles investigados, el mayor porcentaje de influencia fue a partir de la indicación de Cirujanos-Dentistas para los profesionales de la misma área, mientras que académicos y pacientes relataron mayor influencia por parte de la indicación de colegas y propagandas. La publicidad y propaganda interfieren directamente en el uso de estos dentífricos con propuesta aclaradora, siendo la propaganda el mayor incentivador del uso de los dentífricos para pacientes y académicos de odontología. Ya para Cirujanos Dentistas, la influencia fue dirigida por indicación de otro profesional del área, pues se sabe que los agentes contenidos en estos productos sólo eliminan manchas extrínsecas debido a su acción mecánica (abrasión) y el aumento de abrasivos de alto rendimiento como la sílice hidratada, por ejemplo, pueden alterar las características del esmalte, e incluso causar daños al sustrato dental (Azevedo et al., 2008; Horn et al., 2014).

El marketing industrial transmite en los medios de comunicación un número cada vez mayor de productos (cremas y cepillos dentales) disponibles y de precios accesibles a la población, presentando propuesta blanqueadora, que despiertan la atracción de los consumidores (Silva et al., 2015). Los dentífricos con propuesta blanqueadora prometen el aclaramiento dental con resultados satisfactorios con sólo 2 semanas de uso continuo de acuerdo con los fabricantes, generando una curiosidad de las personas, por el fácil acceso y bajo costo (Antonini et al.). En el mercado, es posible encontrar una variedad de dentífricos, con diferentes principios activos e indicaciones clínicas (Martins et al., 2012).

En relación a la interrupción del uso del dentífrico aclarador, aunque no se observan diferencias estadísticamente significativas (Tabla II), se observan diferencias más acentuadas entre los CirujanosDentistas cuando se comparan a los académicos y pacientes, indicando que la mayoría de los pacientes han interrumpido el uso del producto. Entre los motivos relatados para justificar la interrupción y quejas sobre el producto destacan el "sin resultado blanqueador" (32,80 \%) y "sensibilidad dental" (17,90 \%) (Fig. 1). 
La orientación del profesional en cuanto a la elección de ese tipo de dentífrico, así como su utilización, debe ser de vigilancia, para evitar daños en la estructura dental y en los tejidos gingivales. Los dentífricos que contienen agentes aclaradores en sus formulaciones encuentran respaldo en estudios que afirman que la probable pérdida mineral se debe más al propio acto mecánico del cepillado que promueve el desgaste superficial de las unidades dentales favoreciendo la ocurrencia de erosiones y abrasiones, que la acción de los agentes aclaradores propiamente dichos (Worschech et al., 2003).

El verdadero aclaramiento dental requiere modificaciones del color extrínseco, necesitando de alteraciones químicas de la estructura dental que sea perceptible al paciente (Horn et al.). El dentífrico ideal debe ser aquel que promueve la remoción de la mancha extrínseca sin causar el desgaste de la estructura dental, y consecuentemente la sensibilidad dental (Lima et al., 2008). Sin embargo estos dentífricos poseen agentes abrasivos como la sílice, carbonato de calcio y bicarbonato de sodio en su mayor composición y eliminan manchas superficiales, causando en gran parte efectos adversos como descamación e irritación al tejido dental pudiendo producir alteraciones morfológicas irreversibles (Diniz et al., 2005; Antón et al., 2009).

Estudios de microscopia de barrido afirman que el uso de bicarbonato de sodio presente en la composición de los dentífricos no presenta eficacia aclaradora, causan la desmineralización del esmalte, produciendo lesiones de mayor gravedad y el peróxido de carbamida en menor intensidad (Araújo et al.). Siendo así podemos aún presuponer que el tiempo de acción de esos agentes aclaradores al sustrato dental es muy corto para eficacia en su mecanismo de acción, siendo así ineficaz en cuanto a su propuesta blanqueadora, no consiguiendo remover la pigmentación de color amarillo (Antón et al.). Sin embargo, es posible que esa abrasividad pueda generar desgaste excesivo en la superficie del esmalte y dentina, aumentando la porosidad de los tejidos lo que predispone el diente a manchas futuros, y consecuentemente sensibilidad dental en áreas de dentina expuesta entre otros efectos adversos a largo plazo, no produciendo efecto aclarador significativo (Silva et al., 2015).

El presente estudio constató que ninguno de los grupos percibió mejoras significativas en el color de los dientes (Tabla III), siendo los académicos de odontología con mayor percepción no aclaradora de los dentífricos (Fig. 2), y (86,30 \%) no asociaron el uso de dentífricos a otra técnica de aclaramiento dental. Los dentífricos promueven la eliminación de manchas extrínsecas superficiales y pueden entonces, pasar percepción de que ocurrió aclaramiento dental, esta que es entonces utilizada por las propagandas de empresas por cuestiones comerciales (Horn et al.).

Estudios afirman que el uso de diferentes tipos y composiciones de dentífricos aclaradores no promueven el aclaramiento dental, por lo tanto se limita solamente a la remoción mecánica de la pigmentación extrínseca del esmalte con resultados muy similares a los obtenidos con utilización de dentífricos convencionales (Bueno et al., 2011; Torres et al., 2013; Karadas \& Duymus, 2015). Sin embargo otros trabajos afirman que los dentífricos que poseen $1,0 \%$ de peróxido de hidrógeno, $0,243 \%$ de fluoruro de sodio y tripolifosfato de sodio en una base de sílice de alta limpieza, proporciona un nivel estadísticamente significativo de eficacia tanto para el blanqueo dental como para la prevención de manchas extrínsecas, representando una ventaja en comparación con el dentífrico convencional (Sharma et al., 2004), otros estudios concluyeron que el dentífrico puede tener alguna ventaja sobre la pasta convencional en la remoción de manchas, pero la magnitud y la percepción en la diferencia en el color de los dientes parece ser pequeña y de poca relevancia clínica (Moran et al., 2005) esos pueden entonces solamente remover o prevenir manchas superficiales comparado con una pasta de dientes con sílice estándar (Joiner et al., 2008).

En aquellos con propuesta blanqueador son incorporados agentes cosméticos como: abrasivos en mayor tamaño y concentración y/o agentes aclaradores, como ejemplo el peróxido de hidrógeno y de carbamida en pequeñas concentraciones, los abrasivos eliminan manchas extrínsecas y los peróxidos tienen la propiedad de decolorar los dientes al permitir la oxidación de los pigmentos dentales, promoviendo una eliminación química de la mancha extrínseca (Gusmão et al., 2003). Sin embargo, sólo realizan esta función en la concentración adecuada y en el tiempo de acción prolongado para que tenga eficacia, lo que no ocurre durante el cepillado, además, esta asociación de agentes cosméticos causa aumento significativo de la rugosidad del esmalte (Cerqueira et al., 2013).

El proceso químico de aclaramiento dental consiste en una reacción de oxidorreducción, a través 
de la cual la cantidad de pigmentos removidos es proporcional al tiempo de exposición del esmalte al agente aclarador, dentro de límites preestablecidos de mantenimiento de la higiene de las estructuras dentales (Antón et al.). Tanto el proceso de oscurecimiento, como el mecanismo de aclaramiento, sólo es posible gracias a la relativa permeabilidad de las estructuras dentales. Luego, cuanto mayor sea la penetración de la sustancia aclaradora en la intimidad de la unidad dental, mayor será la cantidad de pigmentación que se consigue remover, por lo tanto, mejores resultados estéticos son obtenidos (Lima et al., 2006). La concentración de peróxidos contenidos en los dentífricos es mínima, y el tiempo de acción durante el cepillado bastante reducido, para que se consiga excelencia en la remoción del manchado intrínseco (Soeteman et al.).

La hipersensibilidad fue relatada por los entrevistados entre las quejas, se cree que esta sea debido a su mayor contenido de abrasivos en este tipo de dentífrico causando como efecto adverso la hipersensibilidad (Tabla III). Los dentífricos necesitan tener en su composición sustancias abrasivas como ingredientes para reducir o prevenir manchas extrínsecas, pero esa abrasividad debe ser moderada para evitar la remoción de esmalte (Hilgenberg et al., 2011), en consecuencia podrá causar dolor, incomodidad y sobre todo hipersensibilidad dental y hasta oscurecer los dientes por la disminución del espesor del esmalte por pérdida sustancial, pues la dentina transparenta más, haciendo "oscurecedores" con el paso del tiempo (Tostes et al., 2009).

Con el uso de la Escala Visual Analógica (E.V.A) fue posible percibir el grado de hipersensibilidad dental del producto. La utilización de la Escala Visual Analógica está ampliamente consultada Como instrumentos de búsqueda de fácil interpretación y este puede utilizar cuestionarios para cuantificar la intensidad del dolor (sensibilidad) y su impacto en las actividades del día a día y en la calidad de vida, Además de describir sus demás características clínicas de la hipersensibilidad que se manifiesta de manera incómoda (Godinho et al., 2011; Martinez et al, 2011), estos relataron grado $2(16,40 \%)$ "ligera sensibilidad" y grado $3(9,60 \%)$ "sensibilidad moderada" (Tabla III).

En el cepillado se produce pérdida de la estructura dental del esmalte y esta abrasión aumenta proporcionalmente al tiempo de cepillado (Silva et al., 2015). Así, la abrasividad de un dentífrico puede ser un factor crítico para pacientes con exposición de den- tina radicular que buscan el blanqueo a través de dentífricos, causando hipersensibilidad, arañazos, pliegues, defectos del esmalte y alteraciones en la morfología después del cepillado (Antonini et al.). El uso prolongado de la pasta dental blanqueadora causa el aumento de la rugosidad del esmalte y disminución de la microdureza del esmalte (Rahardjo et al., 2015). Por lo tanto, debe ser utilizado con cautela, debido a su potencial capacidad de aumentar la vulnerabilidad de la superficie del esmalte y, aumentar el riesgo de caries (Watanabe et al., 2005).

El estudio mostró que entre los participantes que indicaron hacer uso de los dentífricos con propuesta aclaradora los más utilizados fueron Colgate Luminous White y Colgate Total 12 (Fig. 3). Estudios muestran que el Colgate Total 12 no fueron efectivos en cuanto a la propuesta blanqueadora, comparando las condiciones inicial y final del color, sin embargo, el Colgate Luminous White ha presentado resultados estadísticamente significativos en la luminosidad a través de la prueba espectrofotómetro Vita Easyshad, se concluye que debido al contenido abrasivo del dentífrico: sílice con alto rendimiento de limpieza y polifosfatos, sin embargo a simple vista el grupo no notó mejora significativa en el blanqueamiento dental (Horn et al.).

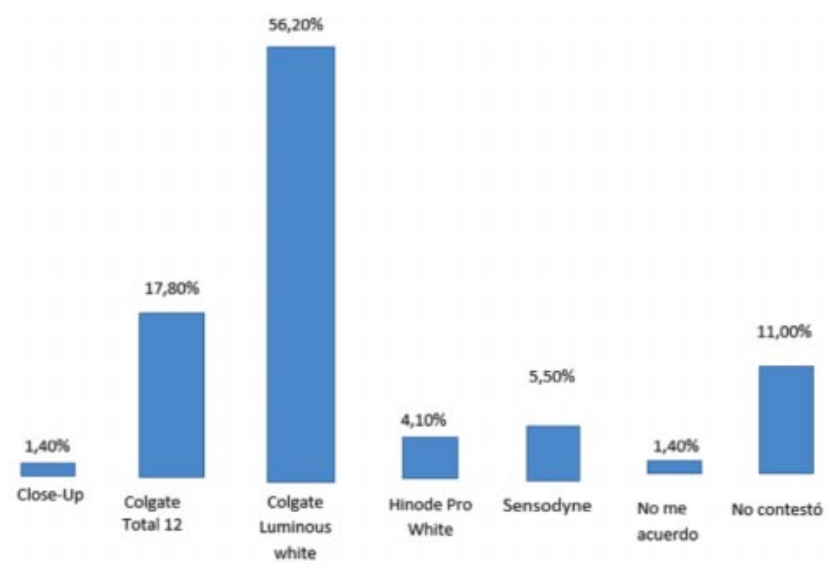

Fig. 3. Identificación de los dentífricos utilizados por los participantes. *Sólo entre los participantes que indicaron hacer uso.

Algunos estudios afirman que no hay diferencia entre la abrasividad del esmalte de las cremas dentales aclaradoras y las cremas dentales comunes, (Joiner et al; Shahabi et al., 2008). Sin embargo, aunque los abrasivos son los componentes presentes en mayor concentración en las cremas dentales, la principal variable abrasiva, no es el tipo exclusivo del abrasivo ni la cantidad, sino las características físicas de los mi- 
nerales, es decir, el tamaño y la forma de las partículas, ya que el carbonato de calcio considerado como uno de los más abrasivos, así como la sílice de menor abrasividad, si se utilizan partículas finas y con formas regulares, se presentaron en las pruebas con baja abrasividad, Cuando se probó lo contrario, fueron más abrasivos (Consani et al.,1995; Andrade Junior et al., 1998; Gusmão et al.; Antonini et al.).

La acción aclaradora de los dentífricos evaluada clínicamente no es probada, pues su tiempo es corto para que haya eficacia en el mecanismo de acción del agente aclarador en el sustrato dental, éstos producen una superficie limpia, pero rugosa y no el aclaramiento propiamente dicho, pudiendo desencadenar la pérdida de la estructura dental (Gusmão et al.; Demarco et al., 2016).

\section{CONCLUSIÓN}

Todos los grupos estudiados hicieron o hacen uso de dentífricos con propuesta aclaradora. La indicación de los dentífricos aclaradores en los grupos de pacientes y académicos se dio por influencia de la propaganda por amplia difusión en los medios de comunicación, sin embargo, para los Cirujanos Dentistas la indicación fue por medio de otros profesionales del área odontológica. Las principales razones para la interrupción del cepillado con dentífricos con propuesta blanqueadora fueron: la presencia de hipersensibilidad dentinaria y la falta de percepción de la eficacia blanqueadora. En consecuencia, los participantes que hayan hecho uso de este producto no lo recomendarían a otras personas. Por lo tanto, estos productos no cumplen la promesa de blanqueo, además del potencial de desencadenar un daño a la estructura dental.

SILVA, A. P. G.; ALVES, L. A. A.; TAVARES, M. A. C. \& FEITOSA, D. A. S. Self-perception of the effects in the use of dentifrices with clarifying proposal. Int. J. Odontostomat., 15(2):484-491, 2021.

ABSTRACT: Aesthetic demands have been increasing in society, it is known that smile is a determining factor in facial aesthetics, and that white teeth are associated with youth sign and oral health. Currently, toothpastes with whitening proposal are within reach of the majority of the population, which ensures a greater demand for these products. However, its effectiveness still raises doubts. This research aimed to evaluate the practice of toothbrushing with toothpastes that have whitening proposal, satisfaction, self-perception of the whitening response and the presence of hypersensitivity caused during its use. The data were collected through questionnaires, in which were evaluated Patients $(n=50)$ attended at the Clinical School of the Centro Universitário Doutror Leão Sampaio, Academics of Dentistry $(n=50)$ of the Centro Universitário Doutror Leão Sampaio and Surgeons-Dentists $(n=50)$ of the city of Juazeiro do Norte, Ceará. Descriptive analysis of frequency distribution was performed, and Pearson's Chi-Square test was performed, adopting a significance level of $5 \%$. It was found that all groups have made or make use of toothpastes with whitening proposal. The advertisement influenced the group of patients and academics of dentistry, however, for the group of Dentists, occurred by the knowledge and influence of another professional Dental Surgeon. Self-perception was negative regarding the whitening efficacy of these products, there was interruption, and the main complaints were dentin hypersensitivity and the non-whitening efficacy, the use of dentifrices with whitening proposal was not recommended by the group studied.

KEY WORDS: aesthetics, dentifrices, oral hygiene, tooth bleaching.

\section{REFERENCIAS BIBLIOGRÁFICAS}

Abdelmegid, F. Y. Effect of whitening toothpastes on bonding of restorative materials to enamel of primary teeth. Niger. J. Clin. Pract., 19 (2):242-7, 2016.

Andrade Junior, A. C. C.; Andrade, M. R. T. C.; Machado, W. A. S. \& Fischer, R. G. Estudo in vitro da abrasividade de dentifrícios. Rev. Odontol. Univ. São Paulo., 12(3):231-6, 1998.

Antón, A. R. S.; Araújo, M. T. B.; Lima, M. J. P. \& Araújo, R. P. C. Estudo in vitro da Ação Clareadora de Dentifrícios. Pesq. Bras. Odontoped. Clin. Integr., 9(1):63-9, 2009.

Antonini, B.; Santos, C. B.; Veloso, K. P. M.; Marchi, G. M.; Rodrigues, J. A. \& Amaral, C. M. Efeito da escovação com dentifrícios clareadores narugosidade superficial do esmalte e da dentina. Rev. Odontol. UNESP, 36(2):121-6, 2007.

Araújo, D. B.; Campos, E. J.; Silva, L. R. \& Araújo, R. P. C. Lesões de esmalte dental relacionadas aos dentifrícios clareadores. Rev. Cienc. Méd. Biol., 8(2):171-81, 2009.

Azevedo, A. M.; Panzeri, H.; Prado, C. J.; De-Mello, J. D. B.; Soares, C. J. \& Fernandes-Neto, A. J. Assessment in vitro of brushing on dental surface roughness alteration by laser interferometry. Braz. Oral Res., 22(1):11-7, 2008.

Bergesch, V.; Aguiar, F. H. B.; Turssi, C. P.; França, F. M. G.; Basting, R. T. \& Amaral, F. L. B. Shade changing effectiveness of plasdone and blue covarine-based whitening toothpaste on teeth stained with chlorhexidine and black tea. Eur. J. Dent., 11(4):432-7, 2017.

Bortolatto, J. F.; Dantas, A. A. R.; Roncolato, A.; Merchan, H.; Floros, M. C.; Kuga, M. C. \& Oliveira Junior, O. B. Does a toothpaste containing blue covarine have any effect on bleached teeth? An in vitro, randomized and blinded study. Braz. Oral Res., 30 (1):1-7, 2016.

Briso, A. L. F.; Rahal, V.; Gallinari, M. O.; Moreira, J. C.; Almeida, L. C. A. G. \& Mestrener, L. R. Análise do clareamento dental caseiro realizado com diferentes produtos: relato de caso. Rev. Odontol. Araçatuba, 35(1):49-54, 2014.

Bueno, F. G.; Lopes, L. G.; Souza, J. B.; Sobrinho, L. C. \& Fonseca, R. B. Influência de diferentes dentifrícios clareadores na cor dental e topografia superficial após ciclos de escovação. Porto Seguro, Sbpcnet, 2011. 
Cerqueira, R. R.; Hofstaetter, F. L.; Rezende, M. ; Martins, G. C.; Loguercio, A. D.; Reis, A. \& Kossatz, S. Efeito do uso de agente dessensibilizante na efetividade do clareamento e na sensibilidade dental. Rev. Assoc. Paul. Cir. Dent., 67(1):64-7, 2013.

Consani, S.; Goes, M. F.; Sinhoreti, M. A. C. \& Sobrinho, L. C. Avaliação "in vitro" da abrasão produzida por dentifrícios fluoretados comerciais. Semin. Cienc. Biol. Saúde., 16(2):308-12, 1995.

Demarco, F. F.; Gluszevicz, A. C.; Mendes, M. L. M.; Nascimento, M. M. C.; Cabreira, N. S. \& Barbieri, V. C. Produtos de autocuidado para clareamento dental. R. F. O., 21(1):143-9, 2016.

Feitosa, D. A. S.; Borges, B. C. D.; Pinheiro, F. H. S. L.; Duarte, R. M.; Araujo, R. E.; Braz, R.; Santos, M. C. M. S. \& Montes, M. A. J. R. Impact of toothbrushing with a dentifrice containing calcium peroxide on enamel color and roughness. Gen. Dent., 63 (1):9-11, 2015.

Godinho, C. J.; Grippi, M. F. \& Costa, L. C. Avaliação clínica do uso de dois novos cremes dentais no tratamento da hipersensibilidade dentinária. R. P. G. Rev. Posgrad., 18(2):72-8, 2011.

Gusmão, E. S.; Melo, J. A. S.; Ramos, C. G.; Santos, R. L.; Araújo, A. C. S. \& Feitosa, D. S. Aplicabilidade Clínica dos Dentifrícios. Int. J. Dent., 2(2):231-5, 2003.

Hilgenberg, S. P.; Pinto, S. C.; Farago, P. V.; Santos, F. A. \& Wambier, D. S. Physical-chemical characteristics of whitening toothpaste and evaluation of its effects on enamel roughness. Braz. Oral Res., 25(4):288-94, 2011

Horn, B. A.; Bittencourt, B. F.; Gomes, O. M. M. \& Farhat, P. A. Clinical Evaluation of the Whitening Effect of Over-the Counter Dentifrices on Vital Teeth. Braz. Dent. J., 25(3):203-6, 2014.

Joiner, A.; Pickles, M. J.; Lynch, S. \& Cox, T. F. The measurement of enamel wear by four toothpastes. Int. Dent. J., 58 (1):23-8, 2008.

Justin, G. A. B.; Dalmolin, I. C.; Cançado, N. M.; Szesz, A. L. \& Martini, E. C. Clinical evaluation of the effectiveness of whitening dentifrices. J. Health Sci., 21(1):82-7, 2019.

Karadas, M. \& Duymus, Z. Y. In vitro evaluation of the efficacy of different over-the-counter products on tooth whitening. Braz. Dent. J., 26(4):373-7, 2015.

Khamverdi, Z.; Kasraie, S. H.; Rezaei-Soufi, L. \& Jebeli, S. Comparison of the effects of two whitening toothpastes on microhardness of the enamel and a microhybride composite resin: an in vitro study. J. Dent. (Tehran), 7(3):139-45, 2010.

Lima, D. A.; Silva, A. L.; Aguiar, F. H.; Liporoni, P. C.; Munin, E.; Ambrosano, G. M. \& Lovadino, J. R. In vitro assessment of the effectiveness of whitening dentifrices for the removal of extrinsic tooth stains. Braz. Oral Res., 22(2):106-11, 2008.

Lima, M. J. P. \& Araújo, R. P. C. Estudo in vitro da ação clareadora do peróxido de hidrogênio a $35 \%$. Rev. Odontol. Cienc., 21(54):376-86, 2006

Maden, E. A.; Altun, C.; Polat, G. G. \& Basak, F. The in vitro evaluation of the effect of xyliwhite, probiotic, and the conventional toothpastes on the enamel roughness and microhardness. Niger. J. Clin. Pract., 21(3):306-11, 2018.

Magalhães, A. C.; Moron, B. M.; Comar, L. P. \& Buzalaf, M. A. R. Uso racional dos dentifrícios. R. G. O. Rev. Gaúcha Odontol., 59 (4):615-25, 2011

Marson, F. C.; Sensi, L. G.; Araújo, F. O.; Monteiro Junior, S. \& Araújo, E. Avaliação clínica do clareamento dental pela técnica caseira. Rev. Dent. Press Estet., 2(4):84-90, 2005.

Martinez, J. E.; Grassi, D. C. \& Marques, L. G. Análise da aplicabilidade de três instrumentos de avaliação de dor em distintas unidades de atendimento: ambulatório, enfermaria e urgência. Rev. Bras. Reumatol., 51(4):299-308, 2011.

Martins, R. S.; Macêdo, J. B.; Muniz, F. W. M. G.; Carvalho, R. S. \& Moreira, M. M. S. M. Composição, princípios ativos e indicações clínicas dos dentifrícios: uma revisão da literatura entre 1989 e 2011. J. Health. Sci. Inst., 30(3):287-91, 2012.

Melo, C. F. M.; Manfroi, F. B. \& Spohr, A. M. Microhardness and roughness of enamel bleached with $10 \%$ carbamide peroxide and brushed with different toothpastes: an in situ study. J. Int. Oral Health, 6(4):18-24, 2014
Menezes, M. M.; Firoozmand, L. M. \& Huhtala, M. F. R. Avaliação do desgaste superficial do esmalte escovado com dentifrícios e submetido à ação de agentes branqueadores. Cienc. Odontol. Bras., 6(1):44-50, 2003.

Monteiro, B. Avaliação In Vitro da Rugosidade Superficial de Resinas Compostas Após Escovação Simulada com Diferentes Dentifrícios. Porto Alegre, Pontifícia Universidade Católica do Rio Grande do Sul, 2014.

Moran, J.; Claydon, N. C.; Addy, M. \& Newcombe, R. Clinical studies to determine the effectiveness of a whitening toothpaste at reducing stain (using a forced stain model). Int. J. Dent. Hyg., 3(1):25-30, 2005.

Pasquali, E. L.; Bertazzo, C. A. \& Anziliero, L. Estudo dos efeitos do clareamento dental sobre o esmalte: uma revisão das evidências para a indicação clínica. Perspectiva, 38(141):99-108, 2014.

Rahardjo, A.; Gracia, E.; Riska, G.; Adiatman, M. \& Maharani, D. A Potential Side Effects of Whitening Toothpaste on Enamel Roughness and Micro Hardness. Int. J. Clin. Prev. Dent., 11(4):23942, 2015.

Roopa, K. B.; Basappa, N.; Prabhakar, A. R.; Raju, O. S. \& Lamba, G. Effect of whitening dentifrice on micro hardness, colour stability and surface roughness of aesthetic restorative materials. J. Clin. Diagn. Res., 10(3):ZC06-11, 2016.

Shahabi, S.; Pesaran, F. \& Kharazifard, M. J. Abrasive properties of three different toothpastes. J. Dent. (Tehran), 5(1):7-11, 2008.

Sharma, N.; Galustians, H. J.; Qaqish, J.; Rustogi, K.; Zhang, Y. P.; Petrone, M. E.; DeVizio, W. \& Proskin, H. M. Comparative tooth whitening and extrinsic tooth stain prevention efficacy of a new dentifrice and a commercially available tooth whitening dentifrice: Six week clinical trial. J. Clin. Dent., 15(2):52-7, 2004.

Silva, J. P. F. L.; Paiva Netto, M. C.; Guimarães, R. P. \& Silva, C. H. V. Avaliação da eficácia de cremes dentais clareadores com uso associado ou não de escova dental especial. Rev. Fac. Odontol. Lins, 21(2):31-9, 2009.

Silva, M. F. R.; Tomo, S.; Oliveira, C. V.; Alves, D. P.; Lima, D. P. \& Pires, H. C. Avaliação in vitro da eficácia de dentifrícios de ação clareadora. Arch. Health Investig., 4(2):35-9, 2015.

Soeteman, G. D.; Valkenburg, C.; Van der Weijden, G. A.; Van Loveren, C.; Bakker, E. \& Slot, D. E. Whitening dentifrice and tooth surface discoloration-a systematic review and meta-analysis. Int. J. Dent. Hyg., 16(1):24-35, 2018.

Torres, C. R.; Perote, L. C.; Gutierrez, N. C.; Pucci, C. R. \& Borges, A. B. Efficacy of mouth rinses and toothpaste on tooth whitening. Oper. Dent., 38(1):57-62, 2013.

Tostes, N. E.; Baptista, N. B.; Carvalho Júnior, O. B.; Francisconi, P. A. S. \& Pires, H. C. Avaliação do desgaste produzido em esmalte por cremes dentais clareadores. Rev. Odontol. Araçatuba, 30(2):9-13, 2009.

Watanabe, M. M.; Rodrigues, J. A.; Marchi, G. M. \& Ambrosano, G. M. In vitro cariostatic effect of whitening toothpastes in human dental enamel-microhardness evaluation. Quintessence Int., 36(6):46773, 2005.

Worschech, C. C.; Rodrigues, J. A.; Martins, L. R. M. \& Ambrosano, G. M. B. In vitro evaluation of human dental enamel surface roughness bleached with $35 \%$ carbamide peroxide and submitted to abrasive dentifrice brushing. Pesq. Odontol. Bras., 17(4):342-8, 2003.

\section{Dirección para correspondencia}

Diala Feitosa

Departamento de Odontología

Centro Universitário Doutor Leão Sampaio

Av. Maria Leticia Leite Pereira s/n - Lagoa Seca

Juazeiro do Norte, CE, CEP 63040-405

BRAZIL

E-mail: dialafeitosa@gmail.com 